\title{
Cannabis Cue Reactivity and Craving Among Never, Infrequent and Heavy Cannabis Users
}

\author{
Erika A Henry*,', Jesse T Kaye², Angela D Bryan', Kent E Hutchison' and Tiffany A Ito' \\ 'Department of Psychology and Neuroscience, University of Colorado, Boulder, Boulder, CO, USA; 'Department of Psychology, University of \\ Wisconsin-Madison, Madison, WI, USA
}

\begin{abstract}
Substance cue reactivity is theorized as having a significant role in addiction processes, promoting compulsive patterns of drug-seeking and drug-taking behavior. However, research extending this phenomenon to cannabis has been limited. To that end, the goal of the current work was to examine the relationship between cannabis cue reactivity and craving in a sample of 353 participants varying in selfreported cannabis use. Participants completed a visual oddball task whereby neutral, exercise, and cannabis cue images were presented, and a neutral auditory oddball task while event-related brain potentials (ERPs) were recorded. Consistent with past research, greater cannabis use was associated with greater reactivity to cannabis images, as reflected in the P300 component of the ERP, but not to neutral auditory oddball cues. The latter indicates the specificity of cue reactivity differences as a function of substance-related cues and not generalized cue reactivity. Additionally, cannabis cue reactivity was significantly related to self-reported cannabis craving as well as problems associated with cannabis use. Implications for cannabis use and addiction more generally are discussed. Neuropsychopharmacology (2014) 39, I214-1221; doi:10.1038/npp.2013.324; published online I8 December 2013
\end{abstract}

Keywords: cannabis; cue reactivity; craving

\section{INTRODUCTION}

Although cannabis is the most widely used illicit drug (Substance Abuse and Mental Health Services Administration, 2010) and is now legal in multiple US states (Colorado Amendment 64, 2012; Washington Initiative 502, 2012), factors that contribute to its continued use in the face of negative consequences are poorly understood (Latimer et al, 2000). One potential explanation for this lack of understanding is the belief that cannabis is a 'soft drug' with minimal addictive properties (Anthony et al, 1994). However, cannabis-dependent individuals suffer a number of adverse side effects (Haney et al, 1999), additional drug-seeking behavior (Kandel, 1975), and neuropsychological decline (Meier et al, 2012). This makes understanding factors that contribute to cannabis dependence important not only to better understand processes underlying cannabis use specifically, but also addiction processes more generally.

One of the defining characteristics of addiction is the process by which drugs alter neural systems involved in motivation and reward, sensitizing brain systems to drugs and their related stimuli. As a result of this process, the perception and mental representation of drug-related

*Correspondence: Dr EA Henry, Department of Psychology and Neuroscience, 345 UCB, University of Colorado, Boulder, CO 80309 0345, USA, Tel: + I 303990 1739, Fax: + I 303492 2967,

E-mail: Erika.Henry@Colorado.edu

Received 2 August 2013; revised II November 2013; accepted 12 November 2013; accepted article preview online 22 November 2013 stimuli are imbued with heightened wanting, or incentive salience, resulting in compulsive patterns of drug-seeking and drug-taking behavior (Robinson and Berridge, 1993). Consistent with this notion, responses to substance-related stimuli are increased among substance users, as reflected in greater attentional engagement (Field and Cox, 2008), autonomic nervous system reactivity (Carter and Tiffany, 1999), and neurophysiological reactivity (Claus et al, 2013; Field et al, 2009; Littel et al, 2012) compared with lighter or non-users. Such cue reactivity is believed to have a causal role in perpetuating substance use and addiction by enhancing substance-related cognitions and subjective craving (Carter and Tiffany, 1999; Littel et al, 2012). In fact, cue reactivity has been associated with important behavioral outcomes such as increased substance consumption and poorer treatment outcomes (Bartholow et al, 2007; Carpenter et al, 2006).

Among the different ways to assess cue reactivity, neurophysiological responses have shown particularly large and consistent effects. Specifically, when substance users view cues related to their substance of choice, the P300 component of the event-related brain potential (ERP) is consistently enhanced across different types of drugs, including alcohol (Bartholow et al, 2007; Namkoong et al, 2004), cocaine (Dunning et al, 2011; Franken et al, 2008), and nicotine (Littel and Franken, 2007). Given the robust association between P300 amplitude and motivational salience (Hajcak et al, 2010), P300 drug-cue-reactivity effects have been interpreted as reflecting engagement of appetitive motivational systems among substance users 
(Littel et al, 2012). Of critical importance, heightened P300 responses among substance users are unique to substancerelated cues, with substance users showing increased P300s to substance-related cues compared with both affectively neutral as well as generally positive stimuli (Bartholow et al, 2010). Thus, increased P300s to substance-related cues does not reflect a generally more reactive motivational system among users, but, rather, selective sensitivity to their substance of use. Moreover, drug-cue-elicited P300s are associated with clinically significant outcomes, including subjective craving and future substance use (Bartholow et al, 2007; Field et al, 2009). A meta-analysis assessing different measures of reactivity indicates that P300 cue reactivity is more strongly related to craving than other indirect measures of attention (eg, reaction time) (Field et al, 2009).

Given the consistency of cue-reactivity effects across different substances of abuse and the general mechanism through which incentive salience is thought to occur, heightened attention to cannabis cues should be expected among heavy cannabis users. In fact, cannabis use has been associated with greater attention to cannabis cues measured behaviorally and with ERPs (Field et al, 2006; Nickerson et al, 2011; Wölfling et al, 2008). However, relative to other substances, cannabis cue reactivity has been studied much less frequently, with few studies examining neurophysiological indications of cannabis cue reactivity. The studies of which we are aware were limited to samples of cannabisdependent individuals (Nickerson et al, 2011; Wölfling et al, 2008), leaving open the question of whether cue reactivity (and, therefore, incentive salience) manifests only after problem levels of cannabis use develop. Moreover, neither past study found a relation between P300 cannabis cue reactivity and craving. This could be due to relatively small sample sizes $(n<15)$ and a restriction in range in craving due to use of drug-dependent samples. Nevertheless, it leaves open the question of whether the cue reactivitycraving link observed for other substances occurs for cannabis.

To understand how these processes operate across a wide range of recreational cannabis use, the current study examines the important questions of whether cannabis use is associated with (1) heightened cue reactivity (assessed via P300 amplitude) and (2) craving among a large sample of participants representing a relatively broad spectrum of use from never to frequent cannabis users. In addition to craving, we also examine how cue reactivity is related to addiction symptoms and problems associated with use. We predict a positive relation between cannabis use and cue reactivity, with P300 amplitudes to cannabis cues increasing linearly with use, and between cue reactivity and craving. Additionally, we expect cue reactivity to correlate positively with use-related problems (eg, symptoms of dependence).

\section{MATERIALS AND METHODS}

\section{Participants}

Participants were 353 University of Colorado students who received monetary compensation in exchange for participation (see Table 1 for sample characteristics). (The total number of participants enrolled was 377. Five participants did not return for their second laboratory session, three participants were dropped from the analysis due to excessive artifact in the ERP data, and ERP data were lost from sixteen participants due to equipment malfunction.). Participants were recruited as never users (ie, must have never tried cannabis), infrequent cannabis users (ie, must use cannabis four times or less per month) and frequent cannabis users (ie, must use cannabis an average of 5 days a week for at least the past year). Data were collected in the first year of a longitudinal study. (One goal of the longitudinal study is to examine trajectories of use over time so we oversampled lighter users.) Only the measures of interest to our current hypotheses will be described in detail, but where appropriate (eg, when they preceded the measures of interest), other measures collected will be noted.

\section{Self-Report Measures}

To evaluate cannabis use, severity, and other substance use, the following questionnaires were administered: Marijuana Dependence Scale (MDS; Stephens et al, 2000), Marijuana Problems Index (MPI; Johnson and White, 1989), and TimeLine Follow Back (TLFB) for quantity and frequency of cannabis, alcohol, and tobacco use (Sobell and Sobell, 1992). Cannabis craving was assessed using an eight-item measure adapted from the Alcohol Urge Questionnaire (Bohn et al, 1995). To assess personality and psychopathology, we administered the Temperament and Character Inventory (TCI; Cloninger et al, 1993), ADHD Current and Childhood Symptom Scales (Willcutt and Bidwell, 2011), and Achenbach System of Empirically Based Assessment (Adult SelfReport Form (ASRF); Achenbach, 2009). See Supplementary Materials for scale descriptions and sample items.

\section{Cue Reactivity Task}

Cue reactivity was assessed by examining P300 amplitudes to infrequent cannabis-related cues (eg, a joint being smoked, a pipe filled with cannabis) as compared with frequent neutral cues in a visual oddball paradigm (eg, Ito et al, 1998). Infrequent exercise pictures were also included to determine the specificity of reactivity to cannabis cues. They were chosen as a control because, like the cannabis pictures, we could include pictures of individuals and groups engaging in the activity, and physical objects associated with the activity. Both cannabis and exercise pictures were shown with equally low probability (6.67\%). Each trial consisted of five images, four of which were neutral. On one-third of the trials, all five pictures were neutral. One of the five pictures was a cannabis picture on another third of the trials and an exercise picture on the remaining third. Within each set of five stimuli, ERPs were recorded during the presentation of a single cannabis, exercise, or neutral target stimulus, which randomly appeared in either the third, fourth, or fifth position in a sequence (Ito et al, 1998). Participants' task was to categorize each image as related to either cannabis (right button press) or exercise (left button press). There were 108 five-sequence trials shown in two equal blocks. For additional task details, see the Supplementary Materials. 
Table I Sample Characteristics of Smoking Groups

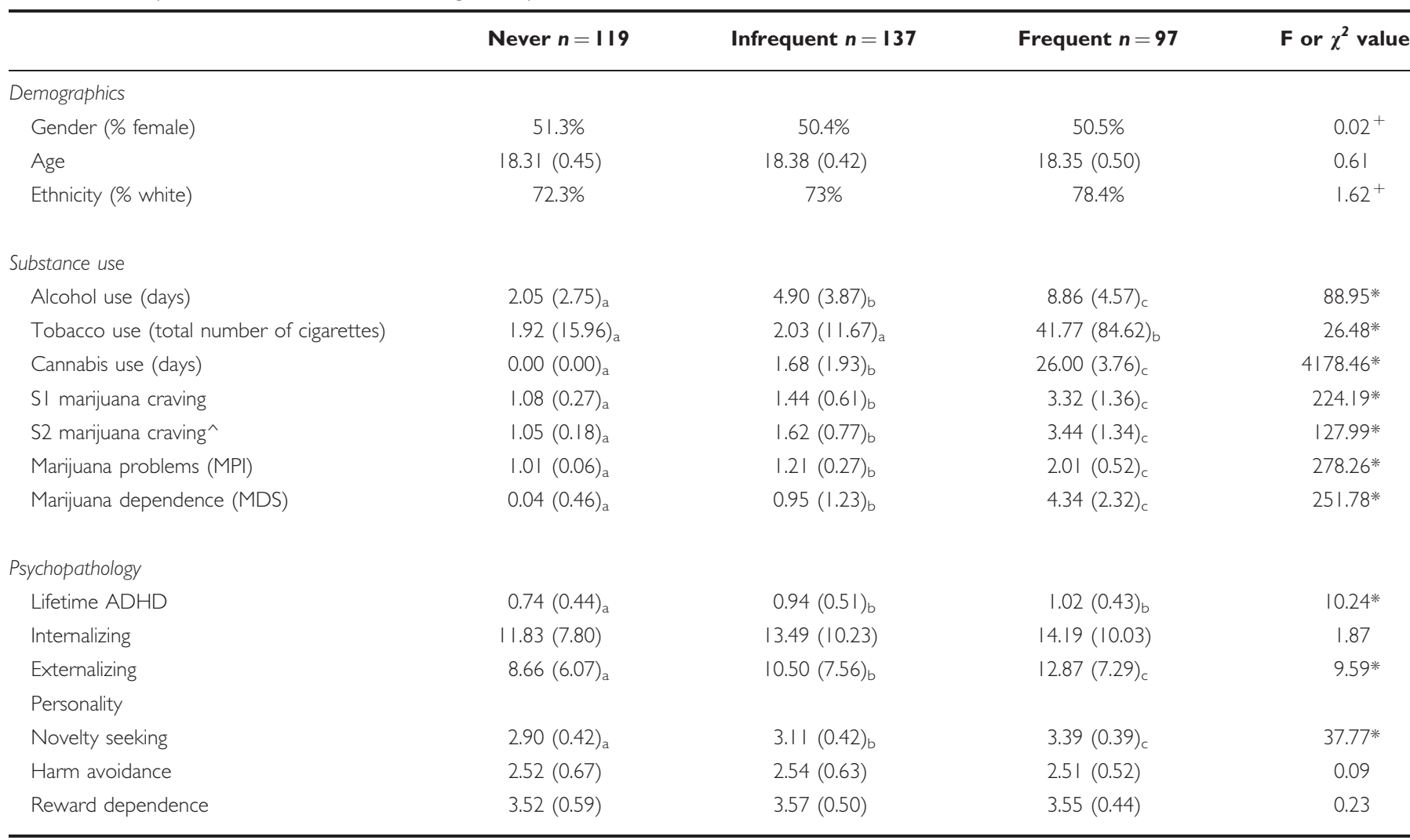

Numbers in parentheses are SD. Alcohol and cannabis use reflect the number of days the substance was used in the 30 days before the first laboratory appointment and so have ranges of $0-30$. Tobacco use reflects the total number of cigarettes smoked in the 30 days before the first laboratory appointment. Other possible ranges are (I) craving I-7, (2) marijuana problems (MPI) I-5, (3) marijuana dependence (MDS) 0-10, (4) lifetime ADHD 0-3, (5) internalizing 0-78, (6) externalizing 0-66, (7) all personality variables I-5. Higher values indicate greater use, craving, problems, dependence, ADHD symptoms, internalizing, externalizing, novelty seeking, harm avoidance, and reward dependence. F values reflect the test of the omnibus Smoking Group main effect. Smoking Group means within the same row with different letter subscripts differ at $p<0.05$, such that different letters denote significant Smoking Group differences. ${ }^{\wedge} N=209 ;{ }^{+}$Indicates the chi-square value with $2 \mathrm{df}$ for the omnibus Smoking Group main effect; $* p<0.001$.

\section{Auditory Oddball Task}

A traditional auditory oddball task was used to further determine the specificity of cue reactivity differences occurring as a function of cannabis use (McFadden et al, 2011). Participants heard infrequent task-relevant tones (oddballs), infrequent task-irrelevant white noise (novel sound) and a frequent context tone (standard), pressing a button when the oddball tone was presented. There were 400 stimuli presented. Both white noise tones and oddball tones were presented with equal probability (12.5\%).

\section{Procedure}

Participants who met criteria for inclusion were scheduled for two laboratory sessions, on average 5.76 days apart $(\mathrm{SD}=5.18)$, and instructed to abstain from alcohol for $24 \mathrm{~h}$, recreational drugs (including cannabis) for $6 \mathrm{~h}$, and caffeine and cigarettes for $1 \mathrm{~h}$ before each laboratory session.

Session 1. After arriving at the lab, participants were provided informed consent and then breathalyzed to ensure a breath alcohol concentration of zero. Adherence to other abstinence requirements was verified verbally. Participants then completed the TLFB and the remaining questionnaires previously described.

Session 2. Participants were breathalyzed and given a urine toxicology screen to insure compliance with our abstinence requirements (we performed the urine test only in the session in which behavioral and electrophysiological data were collected due to financial considerations) then had electrodes affixed to scalp and face locations. Participants then completed two computer tasks not relevant to the present hypotheses. (Participants completed a go/no-go and gambling task (Yeung and Sanfey, 2004) before the auditory oddball task.) Following this, participants engaged in the Auditory Oddball and Cue Reactivity tasks, then completed the Cannabis Craving Questionnaire. (Craving data are available from all participants from session 1, but only available from 209 participants for session 2.)

\section{Electrophysiological Analyses}

P300 analyses were based on mean amplitude values between 450 and $650 \mathrm{~ms}$ post stimulus onset in the Cue Reactivity task and 270 and $400 \mathrm{~ms}$ post stimulus onset in the Auditory Oddball task. P300s in both tasks were initially 

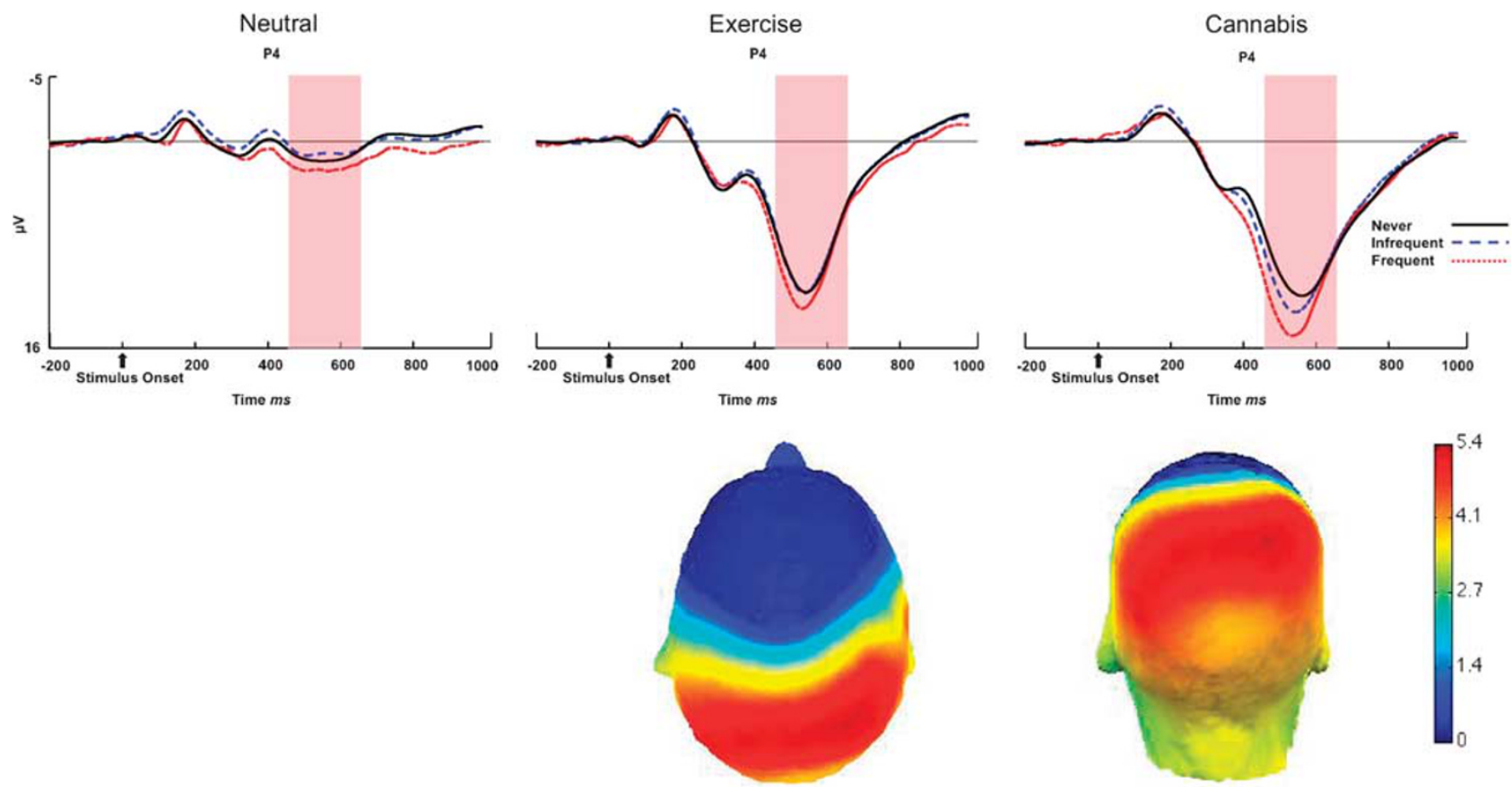

Figure I P300 waveforms elicited by neutral, exercise, and cannabis cues as a function of cannabis use group at P4. Stimulus onset occurred at 0 ms. Three-dimensional presentation of the topographic distribution of ERP activity in the P300 time window from the top and back of the head.

scored at 15 electrodes over midline, left, and right frontal, central, and parietal regions. Consistent with the P300's parietal scalp distribution (eg, Ito et al, 1998), preliminary Lateral $\times$ Sagittal analyses revealed the P300 to be largest at $\mathrm{P} 4$ in the Cue Reactivity task and at $\mathrm{Pz}$ in the Auditory Oddball Task. For simplicity, analyses with each task are reported at these electrodes. (Analyses using the full electrode array, as well as at only $\mathrm{Pz}$ for the Cue Reactivity P300 analyses yielded similar results to those reported at P4). All statistical tests were conducted using two-tailed tests with an alpha criterion level of $p<0.05$.

\section{RESULTS}

\section{Descriptive Statistics}

Table 1 presents basic demographic data and mean values on our measures of substance use, psychopathology, and personality separately for our three cannabis use groups, as well as the results from one-way ANOVAs assessing differences in these constructs as a function of smoking group. In all cases, higher levels of cannabis use were associated with more adverse outcomes (eg, more alcohol use, greater marijuana craving).

\section{Cue Reactivity}

Average response accuracy was high (98\%) and did not vary as a function of smoking group (Supplementary Materials). ERP waveforms are presented in Figure 1. Because of the significant Smoking Group effects in alcohol and tobacco use, ADHD, externalizing problems, and novelty seeking (Table 1), we included these variables as covariates in the current analyses. Although symptoms of internalizing problems did not differ as a function of smoking group
(Table 1), symptoms did directionally increase with use. For completeness, we included internalizing problems in the ANCOVA. In addition, we included gender as a factor in the design as recent research has shown gender differences in reactivity to substance cues (Petit et al, 2013). Thus, we analyzed the data using a 3 (Smoking Group: Never, Infrequent, Frequent) $\times 3$ (Picture: Cannabis, Exercise, Neutral) $\times 2$ (Gender) ANCOVA on P300 amplitudes. (Analyses without covariates produced similar results.) Covariate-adjusted and raw means are presented as a function of smoking group in Table 2.

There was a Picture main effect showing a basic oddball effect, $\mathrm{F}(2,680)=17.33, p<0.001$ (Figure 1 and Table 2). The less frequently presented cannabis and exercise images each elicited larger P300s than the more frequently presented neutral images $(t(352)=45.31$ and 42.02, $p<0.001)$. P300s were also significantly larger to cannabis than the equally low-probability exercise images, demonstrating an overall greater motivational response to cannabis cues across all participants, $t(352)=9.93, p<0.001)$. There was also a Smoking Group main effect, $F(2,340)=3.10$, $p<0.05$, qualified by the expected Smoking Group $\times$ Picture interaction, $\mathrm{F}(4,680)=2.88, p=0.03$. Follow-up analyses revealed greater cue reactivity among cannabis users than never users. Specifically, P300 amplitudes to cannabis cues were significantly larger among frequent cannabis users compared with never users, $t(352)=2.84$, $p=0.005$. Note that these effects occur even when controlling for comorbid alcohol and tobacco use, psychopathology, and novelty seeking. Infrequent cannabis users also showed larger P300 amplitudes to cannabis cues compared with cannabis never users, $t(352)=2.04, p=0.04$. P300s to cannabis cues were not different between frequent and infrequent users, $t(352)=1.54, p=0.12$. Simple Smoking Group effects also occurred for neutral images, with frequent 
cannabis users showing a significantly larger P300 to neutral images compared with infrequent cannabis users $(t(352)=2.62, p<0.01)$ and marginally larger compared with never users $(t(352)=1.92, p=0.06)$. There were no Smoking Group differences in P300 responses to exercise images. No other effects involving Smoking Group were significant. Other a-theoretical effects (eg, Gender main effect) are reported in the Supplementary Materials.

To provide a more powerful test of the relation between levels of use and cue reactivity, we examined correlations between cannabis cue reactivity and a continuous measure of cannabis use (number of days cannabis was used in past 30 days from the TLFB). Cannabis cue reactivity was quantified as the difference in P300s to cannabis as compared with equally infrequent exercise images. As can be seen in Table 3, greater cannabis use was associated with greater cue reactivity.

Cue reactivity, craving, and other symptoms of use. We used the same cannabis cue reactivity contrast score to examine the relation between cue reactivity and craving and

Table 2 Mean ERP Amplitudes for the Cue Reactivity Task as a Function of Smoking Group and Picture

\begin{tabular}{|c|c|c|c|c|}
\hline & \multicolumn{3}{|c|}{ Smoking Group } & \multirow[t]{2}{*}{ Grand mear } \\
\hline & Never & Infrequent & Frequent & \\
\hline \multicolumn{5}{|c|}{ Covariate-adjusted means } \\
\hline Neutral & $1.02(0.37)_{+}$ & $0.83(0.31)_{a}$ & $2.25(0.43)_{b+}$ & 1.37 \\
\hline Exercise & $9.30(0.46)$ & $9.47(0.38)$ & $10.43(0.53)$ & 9.73 \\
\hline Marijuana & $10.23(0.52)_{a}$ & $11.58(0.43)_{b}$ & $12.74(0.60)_{b}$ & 11.52 \\
\hline \multicolumn{5}{|l|}{ Raw means } \\
\hline Neutral & $1.28(0.33)$ & $0.86(0.30)_{a}$ & $1.90(0.36)_{b}$ & 1.34 \\
\hline Exercise & $9.38(0.40)$ & $9.49(0.37)$ & $10.26(0.44)$ & 9.71 \\
\hline Marijuana & $10.23(0.45)_{a}$ & $|1.5|(0.42)_{b}$ & $12.77(0.50)_{c}$ & $|1.5|$ \\
\hline
\end{tabular}

All values are in microvolts. Numbers in parentheses are SD. Covariate-adjusted means show mean P300 amplitudes at mean levels of alcohol and tobacco use, lifetime ADHD, externalizing, internalizing, and novelty seeking. Smoking group means within the same row with different letter subscripts differ at $p \leqslant 0.05$ (ie, different letters denote significant Smoking Group differences). Condition means within the same row differing at $p<0.06$, are denoted with subscript + . other substance use variables. As predicted by incentive salience, cue reactivity was associated with greater craving, although this relation was restricted to craving that was measured immediately after the task and was not present for craving measured in the prior session (Table 3). Greater cue reactivity was also associated with more cannabisassociated problems but unassociated with cannabis dependence symptoms.

\section{Auditory Oddball}

To further confirm that reactivity as a function of cannabis use is specific to cannabis cues, we examined oddball and novelty effects within an auditory oddball task. If the Smoking Group $\times$ Picture interaction in the Cue Reactivity task reflects a specific reactivity to cannabis cues among cannabis users, then the degree of P300 reactivity in the auditory oddball task should not differ as a function of Smoking Group.

ERP waveforms for the Auditory Oddball Task are presented in Figure 2. P300 data were analyzed using a 3 (Smoking Group) $\times 3$ (Tone: Standard, oddball, white noise) $\times 2$ (Gender) ANOVA. (These analyses are based on the 348 participants with usable auditory oddball data.) We obtained oddball and novelty effects that are consistent with extant research, as seen in the Tone main effect, $F(2$, $684)=1307.74, p<0.001$. Both oddball tones $(M=11.98 \mu \mathrm{V})$ and equally infrequent white noise $(M=12.47 \mu \mathrm{V})$ elicited significantly larger P300 amplitudes compared with the Standard tones $(M=0.71 \mu \mathrm{V} ; t(347)=38.32$ and 47.60 , $p<0.001)$. P300s were larger to the novel white noise than oddball tones, $t(347)=2.10, p<0.05$. Of importance, none of the effects were moderated by smoking group (other significant a-theoretical effects are reported in the Supplementary Materials).

\section{DISCUSSION}

The current study investigated whether differences in cannabis use among never users, infrequent users, and frequent users result in the biased processing of cannabis cues. Of primary importance, although cannabis is considered to be minimally addictive (Anthony et al, 1994), we see a clear cue-reactivity effect in which P300s to cannabis cues were significantly larger among frequent cannabis

Table 3 Correlations between Cue Reactivity, Cannabis Use and Addiction Constructs

\begin{tabular}{|c|c|c|c|c|c|c|}
\hline & $\begin{array}{c}\text { Cannabis cue } \\
\text { reactivity }\end{array}$ & Cannabis use & $\begin{array}{c}\text { Dependence } \\
\text { symptoms }\end{array}$ & $\begin{array}{l}\text { Cannabis } \\
\text { problems }\end{array}$ & Craving (sI) & Craving (s2) \\
\hline Cannabis use & $0.18 * *$ & & & & & \\
\hline Cannabis problems & $0.12 *$ & $0.78 * *$ & $0.87 * * *$ & & & \\
\hline
\end{tabular}

Cannabis cue reactivity is represented by the difference in P300 amplitude to cannabis and exercise images. Cannabis use =total number of days cannabis was consumed 30 days prior to session I. Dependence symptoms = Marijuana Dependence Scale. Cannabis problems= Marijuana Problems Index. sI = session I; $s 2=$ session 2. All $n=353$ except $s 2$ craving, where $n=209$. $* p<0.05$, *** $p<0.01$. 

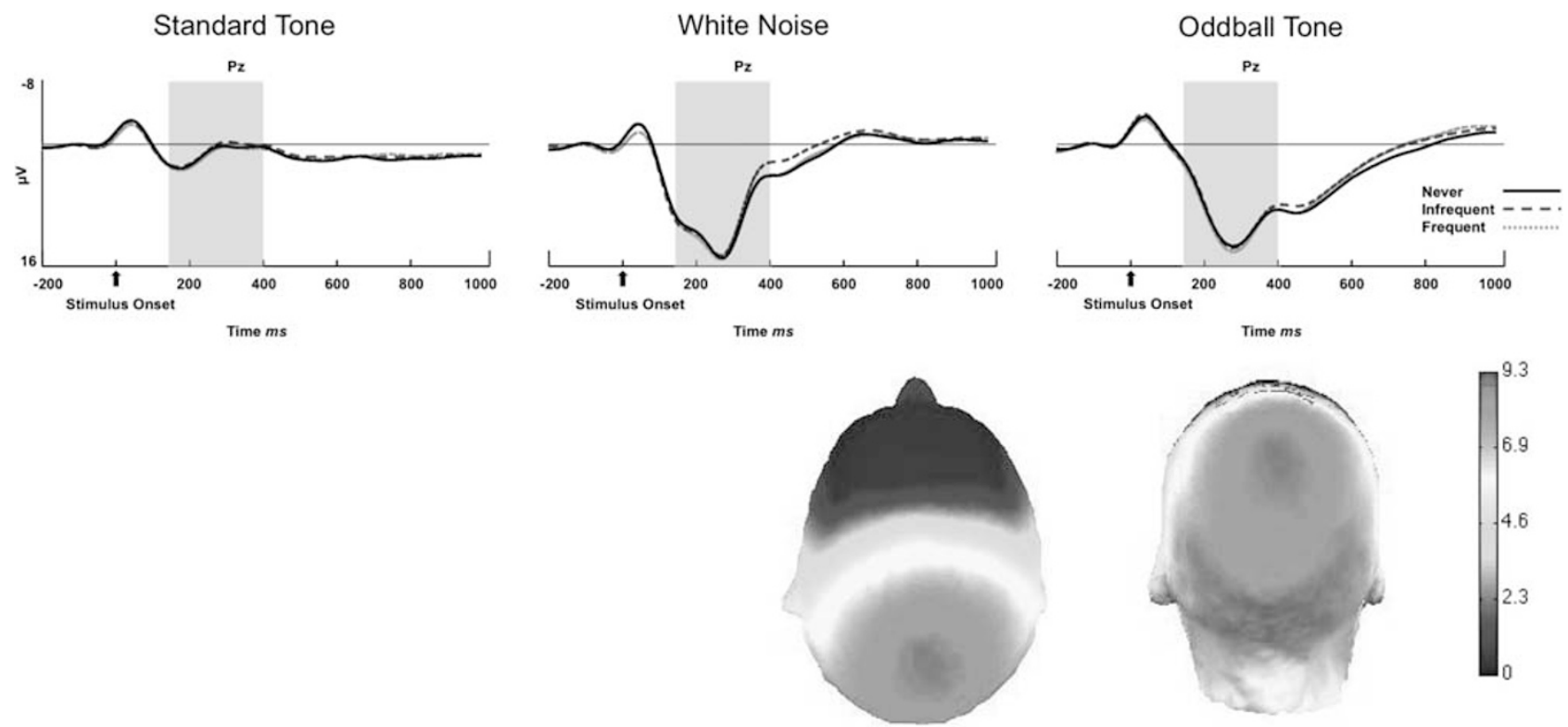

Figure 2 P300 waveforms elicited by standard tones, white noise tones, and oddball tones as a function cannabis use group at Pz. Stimulus onset occurred at 0 ms. Three-dimensional presentation of topographic distribution of ERP activity in the P300 window from the top and back of the head.

users compared with never users. Past studies have shown a P300 cue-reactivity effect, but only tested dependent users (Nickerson et al, 2011; Wölfling et al, 2008). Our results are thus the first to demonstrate that cue reactivity, and the sensitization of reward systems to substance-related cues it is thought to represent, is not limited to individuals who have developed problem levels of use, but rather is a process that becomes intensified with continued and/or more frequent use. Even infrequent users, defined as those who use four times or less per month, show significant cannabis cue reactivity, and our analyses using a continuous measure of use (number of days used), show a linear relation between use and reactivity. The current work is also the first to demonstrate that cannabis cue reactivity occurs even when controlling for alcohol and tobacco use, psychopathology, and novelty seeking, all of which varied as a function of cannabis use group. These effects occurred equally for both male and female users. Importantly, no concomitant relation between P300 amplitude and use was seen to equally rare exercise pictures in the Cue Reactivity task or rare tones in the Auditory Oddball task.

Increased P300s are not specific to cannabis cues, or substance cues more generally. In fact, the very reason the P300 is used as a marker of cue reactivity is because it robustly increases to motivationally relevant stimuli (eg, food, erotica, conspecifics). This motivational response is not necessarily pathological in and of itself, but can be problematic when elicited by cues associated with problem behavior. In support of this, cue reactivity was associated with both craving and problems associated with cannabis use.

Notably, although our results demonstrate that there is indeed a relationship between cannabis cue reactivity and craving, this effect only manifested when craving was measured in close temporal proximity to cue reactivity (ie, after the Cue Reactivity task rather than days before). The temporal specificity of the reactivity-craving relation shows that although the cue-reactivity effect is related to relatively stable variation in cannabis use (ie, use over the past 30 days in the case of the TLFB, as well as the initial classification of use made by our categorical classification of use), the reactivity-craving link is more situational. While research has demonstrated a relationship between cue reactivity and subsequent craving (Carter and Tiffany, 1999), to our knowledge this is the first work to assess the relationship between craving and cue reactivity on separate days. While this finding is preliminary, it could have important implications for understanding the cravingreactivity link and how it relates to future cannabis use.

Perhaps not surprising given the link between cue reactivity and level of use, cue reactivity was also associated with experiencing more problems in life due to cannabis use. By contrast, cue reactivity did not relate to dependence. The lack of relationship with dependence could be due to use of a self-report measure of dependence (as opposed to a clinical interview) or to the relatively low level of reported dependence. Although our frequent users reported using cannabis nearly every day in the past month $(M=26$ days), their mean level of self-reported dependence symptoms was below the scale midpoint.

There are some limitations to consider. First, although these results are the first to demonstrate a link between cannabis cue reactivity and craving, the relationship was modest in size. Our participants were asked to abstain from cannabis for only $6 \mathrm{~h}$ before their laboratory session; it is possible that a longer abstinence period would result in greater levels of craving, and thus, a stronger relationship with cannabis cue reactivity. It is also possible that because cannabis does not elicit addictive symptoms as severe as other substances (Anthony et al, 1994), the relationship between cannabis cue reactivity and craving will necessarily be modest. Nevertheless, the evidence of the cue reactivitycraving link suggests that cannabis use does sensitize brain reward pathways to cannabis-related stimuli in ways similar to other substances of abuse.

Although participants were asked to abstain for cannabis use for $6 \mathrm{~h}$ before their laboratory session, there may have 
still been cannabinoids in their system. Acute effects of cannabis, however, peak $\sim 30 \mathrm{~min}$ after consumption (Grotenhermen, 2003) and impairment usually lasts for only 2-3 h (Crean et al, 2011), arguing against any impairment during testing. Consistent with this, we saw no differences in task performance as a function of use. Moreover, we conducted post-hoc analyses to ensure that time since last use did not influence the Cannabis P300, seeing no relationship between number of hours since participants smoked and the P300 cannabis-exercise difference score $(r=-0.05, p=0.61)$. Finally, acute cannabis ingestion has been shown to decrease P300 amplitude (D'Souza et al, 2012), suggesting that any cannabinoids in the system would actually work against our hypotheses of a selective increase in P300s as a function of cannabis use. We do also note that abstinence was verified only with self-report. Participants' readiness to report on other aspects of their substance use coupled with the absence of overall very high levels of performance leads us to expect the data are reliable. We also administered a urine toxicology test before the ERP tasks. Because participants had no way of knowing how sensitive the test was, it is reasonable to assume they thought the test could detect compliance with the abstinence requirements. Nevertheless, verification with more objective measures would be desirable in future research.

We also presently cannot determine why P300s were larger to neutral pictures among frequent as compared with infrequent users. It could reflect some idiosyncratic categorization of the stimuli (eg, rather than seeing all the stimuli as neutral, frequent users may have found some of the stimuli more affectively arousing). Future research might examine if cannabis use affects basic emotional processing. This unexpected effect, however, does not alter our conclusions. When we compare P300s to cannabis and exercise imagesthe most appropriate contrast because both were shown with equal frequency-we see that cannabis use correlates with cue reactivity. Moreover, ancillary analyses correlating level of use with the difference in P300 amplitude to cannabis as compared with neutral images shows a similar increase as a function of use $(r=0.13, p<0.02)$.

\section{Implications}

The wide spread use and increasing legalization of cannabis stands in stark contrast with the relative dearth of research on the basic mechanisms that explain its use, especially in comparison with what is known about other substances of abuse. Sensitization of reward pathways have been argued to explain compulsive drug-seeking and taking for other substances, but popular beliefs that cannabis is minimally addictive raise questions about whether the same mechanisms govern cannabis use. Our results suggest they do by demonstrating heightened motivational responses to cannabis cues among cannabis users. This is important for our understanding of substance use in general, suggesting that the same processes of incentive sensitization governing other substances of abuse also apply to cannabis use. It also has specific implication for cannabis use. Whereas other demonstrations of cannabis cue reactivity were confined to only dependent users, our results suggest that sensitization of reward systems to substance-related cues occurs even at lower levels of recreational use. The correlation in our work between cue reactivity and craving, and demonstrations in other contexts that substance cue reactivity prospectively predicts future use (Bartholow et al, 2007) suggest that P300 cue reactivity may be an important endophenotype for future substance use. Given the current cultural milieu of marijuana legalization and increasing use among adolescents (National Institute on Drug Abuse, 2013), such an endophenotype that can identify compulsive wanting may be of particular importance.

\section{FUNDING AND DISCLOSURE}

The authors declare no conflict of interest.

\section{ACKNOWLEDGEMENTS}

This research was supported by a grant from the National Institute on Drug Abuse (R01 DA024002, Tiffany Ito PI).

\section{REFERENCES}

Achenbach TM (2009). The Achenbach System of Empirically Based Assessment (ASEBA): Development, Findings, Theory, and Applications. University of Vermont Research Center for Children, Youth, and Families: Burlington, VT.

Anthony JC, Warner LA, Kessler RC (1994). Comparative epidemiology of dependence on tobacco, alcohol, controlled substances, and inhalants: Basic findings from the National Comorbidity Survey. Exp Clin Psychopharmacol 2: 244-268.

Bartholow BD, Henry EA, Lust SA (2007). Effects of alcohol sensitivity on $\mathrm{P} 3$ event-related potential reactivity to alcohol cues. Psychol Addict Behav 21: 555-563.

Bartholow BD, Lust SA, Tragesser S (2010). Specificity of P3 eventrelated potential reactivity to alcohol cues in individuals low in alcohol sensitivity. Psychol Addict Behav 24: 220-228.

Bohn MJ, Krahn DD, Staehler BA (1995). Development and initial validation of a measure of drinking urges in abstinent alcoholics. Alcohol Clin Exp Res 19: 600-606.

Carpenter KM, Schreiber E, Church S, McDowell D (2006). Drug Stroop performance: relationships with primary substance of use and treatment outcome in a drug-dependent outpatient sample. Addict Behav 31: 174-181.

Carter BL, Tiffany ST (1999). Meta-analysis of cue-reactivity in addiction research. Addiction 94: 327-340.

Claus ED, Blaine SK, Filbey FM, Mayer AR, Hutchison KE (2013). Association between nicotine dependence severity, BOLD response to smoking cues, and functional connectivity. Neuropsychopharmacology 38: 2363-2372.

Cloninger CR, Svrakic DM, Przybeck TR (1993). A psychobiological model of temperament and character. Arch Gen Psychiatry 50: $975-970$.

Colorado Amendment 64 (2012). Amendment 64: Use and Regulation of Marijuana http://www.colorado.gov/cs/Satellite? blobcol=urldata\&blobheader=application $\% 2$ Fpdf\&blobkey=id \&blobtable $=$ MungoBlobs \&blobwhere $=1251834064719 \&$ ssbinary $=$ true.

Crean RD, Crane NA, Mason BJ (2011). An evidence based review of acute and long-term effects of cannabis use on executive cognitive functions. J Addict Med 5: 1-8.

Dunning JP, Parvaz MA, Hajcak G, Maloney T, Alia-Klein N, Woicik PA et al (2011). Motivated attention to cocaine and emotional cues in abstinent and current cocaine users - an ERP study. Eur J Neurosci 33: 1716-1723.

D'Souza DC, Fridberg DJ, Skosnik PD, Williams A, Roach B et al (2012). Dose-related modulation of event-related potentials to 
novel and target stimuli by intravenous delta super(9)-THC in humans. Neuropsychopharmacology 37: 1632-1646.

Field M, Cox WM (2008). Attentional bias in addictive behaviors: a review of its development, causes, and consequences. Drug Alcohol Depend 97: 1-20.

Field M, Eastwood B, Bradley BP, Mogg K (2006). Selective processing of cannabis cues in regular cannabis users. Drug Alcohol Depend 8: 75-82.

Field M, Munafo MR, Franken IHA (2009). A meta-analytic investigation of the relationship between attentional bias and subjective craving in substance abuse. Psychol Bull 135: 589-607.

Franken IHA, Dietvorst RC, Hesselmans M, Franzek EJ, van de Wetering BJ, Van Strien JW (2008). Cocaine craving is associated with electrophysiological brain responses to cocaine-related stimuli. Addict Biol 13: 386-392.

Grotenhermen F (2003). Pharmacokinetics and pharmacodynamics of cannabinoids. Clin Pharmacokinet 42: 327-360.

Haney M, Ward AS, Comer SD, Foltin RW, Fischman MW (1999). Abstinence symptoms following smoked marijuana in humans. Psychopharmacology 141: 395-404.

Hajcak G, MacNamara A, Olvet DM (2010). Event-related potentials, emotion, and emotion regulation: an integrative review. Dev Neuropsychol 35: 129-155.

Ito TA, Larsen JT, Smith NK, Cacioppo JT (1998). Negative information weighs more heavily on the brain: The negativity bias in evaluative categorizations. J Personality Social Psychol 75: 887-900.

Johnson V, White HR (1989). An investigation of factors related to intoxicated driving behaviors among youth. J Studies Alcohol 50: 320-330.

Kandel D (1975). Stages in adolescent involvement in drug use. Science 190: 912-914.

Latimer WW, Winters KC, Stinchfield R, Traver RE (2000). Demographic, individual, and interpersonal predictors of adolescent alcohol and marijuana use following treatment. Psychol Addict Behav 14: 162-173.

Littel M, Euser AS, Munafo MR, Franken IHA (2012). Electrophysiological indices of biased cognitive processing of substance-related cues: A meta-analysis. Neurosci Biobehav Rev 36: 1803-1816.

Littel M, Franken IHA (2007). The effects of prolonged abstinence on the processing of smoking cues: An ERP study among smokers, ex-smokers and never-smokers. J Psychopharmacol 21: 873-882.

McFadden KL, Healy KM, Dettmann ML, Kaye JT, Ito TA, Hernandez TD (2011). Acupressure as a non-pharmacological intervention for traumatic brain injury (TBI). J Neurotrauma 28: $1-14$.

Meier MH, Caspi A, Ambler A, Harrington H, Houts R, Keefe RS et al (2012). Persistent cannabis users show neuropsychological decline from childhood to midlife. Proc Natl Acad Sci USA 109: 15970-15971.

Namkoong K, Lee E, Lee CH, Lee BO, An SK (2004). Increased P3 amplitudes induced by alcohol-related pictures in patients with alcohol dependence. Alcohol Clin Exp Res 28: 1317-1323.

National Institute on Drug Abuse (2013). Adolescent cigarette, alcohol use declines as marijuana use rises http://www. drugabuse.gov/news-events/nida-notes/2013/02/adolescent-cigarette-alcohol-use-declines-marijuana-use-rises.

Nickerson LD, Ravichandran C, Lundahl LH, Rodolico J, Dunlap S, Trksak GH et al (2011). Cue reactivity in cannabis-dependent adolescents. Psychol Addict Behav 25: 168-173.

Petit G, Kornreich C, Verbanck P, Campanella S (2013). Gender differences in reactivity to alcohol cues in binge drinkers: A preliminary assessment of event-related potentials. Psychiatry Res 209: 494-503.

Robinson TE, Berridge KC (1993). The neural basis of drug craving: An incentive-sensitization theory of addiction. Brain Res Rev 18: 247-291.

Sobell LC, Sobell MB (1992). Timeline Follow-back: A technique for assessing self-reported ethanol consumption. In Allen J, Litten RZ (eds) Measuring Alcohol Consumption: Psychosocial and Biological Methods. Humana Press: Totowa, NJ, pp 41-72.

Stephens RS, Roffman RA, Curtin L (2000). Comparison of extended versus brief treatments for marijuana use. J Consult Clin Psychol 68: 898-908.

Substance Abuse and Mental Health Services Administration (2010). Results from the 2009 National Survey on Drug Use and Health. Department of Health and Human Services: Rockville.

Washington Initiative 502 (2012). Summary of Initiative $502 \mathrm{http}: / /$ www.leg.wa.gov/House/Committees/OPRGeneral/Documents/ 2012/I-502\%20summary.pdf.

Willcutt EG, Bidwell LC (2011). Etiology of ADHD: implications for assessment and treatment. In Hoza BEvans SW (eds) Treating Attention Deficit Hyperactivity Disorder. Civic Research Institute: Kingston, NJ, pp 6-2-6-18.

Wölfling K, Flor H, Grüsser SM (2008). Psychophysiological responses to drug associated stimuli in chronic heavy cannabis use. Eur J Neurosci 27: 976-983.

Yeung N, Sanfey AG (2004). Independent coding of reward magnitude and valence in the human brain. J Neurosci 24: 6258-6264.

Supplementary Information accompanies the paper on the Neuropsychopharmacology website (http://www.nature.com/npp) 